\title{
TOOCC: Enabling Heterogeneous Systems Interoperability in the Study of Energy Systems
}

\author{
Brígida Teixeira, Francisco Silva, Tiago Pinto, Gabriel Santos, Zita Vale \\ GECAD - Knowledge Engineering and Decision Support Research Center \\ Institute of Engineering - Polytechnic of Porto (ISEP/IPP) \\ Porto, Portugal \\ \{bccta, fspsa, tmcfp, gajls, zav $\} @$ isep.ipp.pt
}

\begin{abstract}
The environmental impact and the scarcity of limited fossil fuels led to the need of investment in energy based on renewable sources. This has driven Europe to implement several policies that changed the energy market's paradigm, namely the incentive to microgeneration. The penetration of energy sources from intermittent nature has increased the unpredictability of the system, which makes simulation and analysis tools essential in order to provide decision support to entities in this sector. This paper presents the Tools Control Center (TOOCC) as a solution to increase the interoperability between heterogeneous agent-based systems, in the energy field. The proposed approach acts as a facilitator in the interaction between different systems through the usage of ontologies, allowing them to communicate in the same language. To understand the real applicability of this tool, a case study is presented concerning the interaction between several systems, with the purpose of enabling the energy resource scheduling of a microgrid, and the reaction of a house managed by a house management system.
\end{abstract}

Index Terms-- Agent-Based Systems, Demand Response, Energy Efficiency, Ontologies, Systems Interoperability.

\section{INTRODUCTION}

The intensive exploitation of fossil based energy resources led to the emergence of very challenging problems for today's society, particularly due to environmental impact and scarcity of these resources. Consequently, the need for investment in energy from renewable sources (e.g.: solar, wind, water) arises in order to reduce the emission of greenhouse gases, ultimately leading to an increased life quality and sustainable growth [1]. Additionally, the use of this type of energy also allows greater energy independence, lower costs and reduced environmental impact.

In the last decade, the European Union (EU) has established several policies that contributed to fomentation of investment and change of legislation in this field, leading to large-scale implementation of microgeneration [2]. As result, the energy sector paradigm has changed completely, becoming more complex and dynamic in the production, distribution, storage and consumption of energy. In this context, Smart

This work has received funding from the European Union's Horizon 2020 research and innovation programme under the Marie Sklodowska-Curie grant agreement No 641794 (project DREAM-GO) and from FEDER Funds through COMPETE program and from National Funds through FCT under the project UID/EEA/00760/2013
Grids (SG) [3], Demand Response (DR) and consumer empowerment concepts emerge [4].

Through data collected from multiple sensors installed along the power grid, SG enable analyzing and processing information in order to make decisions and act automatically in the system, with the goal of making it more efficient, reliable and sustainable. From the network operators' point of view, it is possible to make strategic forecasts that enable adequate DR, which allows to minimize costs, reduce energy waste, control and plan energy supply and prevent failures [5]. Consumers, through Smart Meters (SM), can be aware of their consumption profile, in real time. Therefore, it is easier to track costs, choose other energy price tariffs, change services, or schedule the usage of appliances. Furthermore, the consumer can take the prosumer role, by connecting to the network as a low-voltage energy producer, by photovoltaic or wind generators (microgeneration).

The use of simulation and analysis tools and the study of different market mechanisms and relationships among its stakeholders becomes essential in this context. However, one of the main challenges in this area is the development of tools to support the decision and address the problem with capability to handle the inherent complexity in this scope [6].

This paper proposes the Tools Control Center (TOOCC), which has the objective of increasing the interoperability between heterogeneous agent-based systems [7] for the study of power and energy systems, e.g. electricity markets, SG operation, energy management. TOOCC acts as a facilitator in the interaction between entities/agents with distinct natures, from various systems, through the use of ontologies [8].

After this introductory section, the proposed tool is presented in Section II, with emphasis on the main steps required for the simulation. Section III presents the case study and the main results, which show the benefits of this tool. The main conclusions of this work are presented in Section V.

\section{TOOCC}

The Tools Control Center (TOOCC) is a multi-agent [9] tool that acts as a central facilitator, enabling the creation, execution/simulation and analysis of study scenarios in the power and energy systems field. For this, TOOCC is integrated with other multi-agent systems, which allows the construction of complex scenarios, depending on the number 
of interconnected systems. Thus, the user can obtain independent results for the algorithms or tools, or launching scenarios to converge these tools in a single unified system society of agent-based systems.

For each desired scenario, a software agent is created to ensure the communication with external systems, which provides the necessary services for the respective scenario. These agents are created and managed by a central agent, which is responsible for scenarios setup, monitoring of children agents' execution process and, subsequently, analyzing the results. This tool has a task distribution mechanism for the domain machines equipped with the necessary software and properly configured for this purpose. This feature allows the construction of scenarios without the need to install the required software in the user's computer given that this system is able to detect the machines that fulfill the requirements of the various tasks of the agents.

Figure 1 presents the global overview of TOOCC.

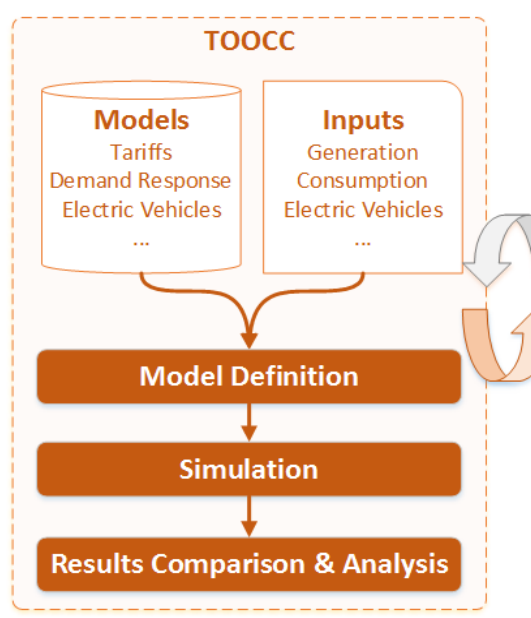

Figure 1. TOOCC overview

From Figure 1, it can be seen that the scenarios' execution involves three main phases. Firstly, scenarios are defined by using stored models and input data, which are necessary for the algorithm/tool execution. After this step, the scenarios are executed, interacting with other external tools. Finally, the user can compare and analyze the achieved results. The following subsections present the description of each step.

\section{A. Models and Inputs}

In order to configure the scenarios' execution, it is necessary to consider the input data expected by different external systems. Each system has its own requirements and models that must be filled out so that they can perform. For example, the configuration settings for the smart grid tools consider data from distributed generators, storage units, loads, and electric vehicles, among other [10].

In addition, other models can be included in the scenario, in order to complete it: e.g. DR programs [4]; aggregation models and interaction between players from energy market and power consumption tariffs. The diversity of supported models enables setting up different scenarios to study their impact on simulations.

\section{B. Model Definition}

Using the several available models and input data, users can setup the required scenarios. Scenarios can be related to the simulation of an individual algorithm, or the integration of multiple algorithms and/or tools for the development of a more complex scenario. The required models and inputs that are necessary to execute the desired algorithm/tool are considered. It is also possible to setup multiple scenarios simultaneously, getting the results for each independently.

\section{Simulation}

The following step of the process consists in the execution of configured algorithms and tools. For this purpose, TOOCC communicates with several external multi-agent systems [7]. These agent-based tools communicate with each other using ontologies, which enables the interoperability between different systems, by making sure all entities "speak the same language" and understand the same concepts and terms in the same way, thereby preventing different interpretations of the same information. The use of ontologies also enables the interaction with external systems, thus facilitating the execution of numerous diverse systems and algorithms in combined simulations. Figure 2 shows the interaction between the currently considered systems (TOOCC is not limited to these systems, being open to the integration of many others).

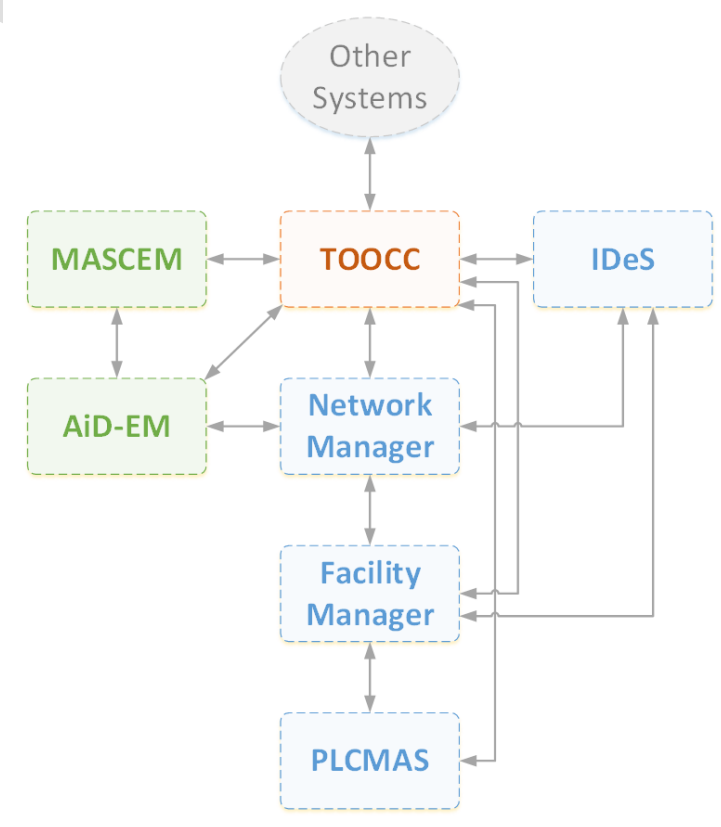

Figure 2. TOOCC interaction with multiple multi-agent systems

In Figure 2 the following systems can be seen:

- Intelligence and Decision Support multi-agent system (IDeS) - Executes the different DR, optimization, scheduling, forecasting, and decision support algorithms;

- Multi-Agent System for Competitive Electricity Markets (MASCEM) [11] - Runs electricity market simulations; 
- Adaptive Decision Support for Electricity Market Negotiation (AiD-EM) [12] - Provides intelligent support for player's decisions in electricity market negotiations;

- Network Manager (NM) [10] - Enables the energy management for a grid (Smart/Micro);

- Facility Manager (FM) [13] - Manages facilities' energy resources;

- Programmable Logic Controller Multi-Agent System (PLCMAS) - Controls the physical devices connected to any PLC (e.g. lights, sockets, HVAC);

- Other Systems - External tools which can provide new services or functions for TOOCC.

\section{Results Comparison and Analysis}

Finally, the last step corresponds to the analysis and comparison of results. The results of the scenarios' execution are presented to the user, in the form of graphs and tables. Moreover, it is also possible to directly compare different scenarios and analyze their results through auxiliary graphics.

\section{CASE STUDY}

This section presents a case study designed to illustrate the use of TOOCC to set up a complex scenario. For this purpose, the integration of several systems is made to combine their results and achieve a more complete, robust and reliable result.

Thereafter, the scope of this case study is described, including the definition of the constitution and characteristics of the considered grid, and of a residence that is used as study subject. After this, a description is given about how TOOCC is able to simulate the case study using the available tools. Finally, the simulation's results are presented.

\section{A. Context}

The developed scenario simulates the energy scheduling of a real 25-bus microgrid, where one of the bus corresponds to a residence accommodated in the GECAD's laboratory. Furthermore, the residence must schedule its own energy management plan, considering the fulfillment of its needs as well as the network operator's needs. After that, the house management system can apply the changes in its physical devices, taking advantage of the obtained results.

The considered microgrid has 25 BUS, 90 loads, 17 PV systems, 17 storage systems and 5 external suppliers. Its data set is composed with data from GECAD (Portugal) and from Discovergy (Germany). The consumers are residential houses, residential buildings, office buildings and a residential heat pump. Figure 3 shows the scheme of the grid, highlighting the load that corresponds to the considered residence.

Load 6 corresponds to the considered residential house, composed by a bedroom, a hall, a bathroom, a kitchen and a living room. It is, in reality, a laboratory that aims to simulate the energy consumption of a house, by monitoring the usage of several appliances and loads, namely HVAC, lights, sockets, among others. The data used in this case study can be consulted in [14].

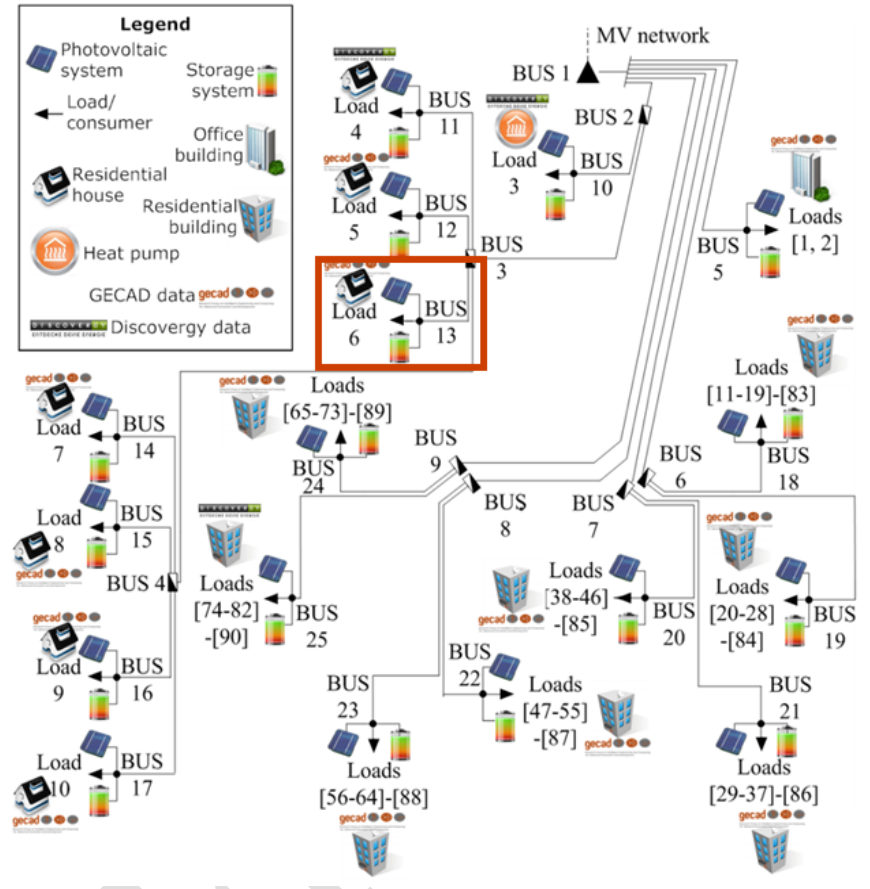

Figure 3. 25-bus microgrid scheme

\section{B. TOOCC Simulation}

In order to simulate the case study presented in $A$, TOOCC integrates several systems, namely NM, FM, the IDeS and PLCMAS. Thereby, it is necessary to define the scenario in order to achieve the desired objectives. The used configuration is just an example of what it is possible using TOOCC, since the algorithms that are described in this paper can interact with TOOCC in many ways. In this case, the setting is configured to correspond to the diagram illustrated in Figure 4.

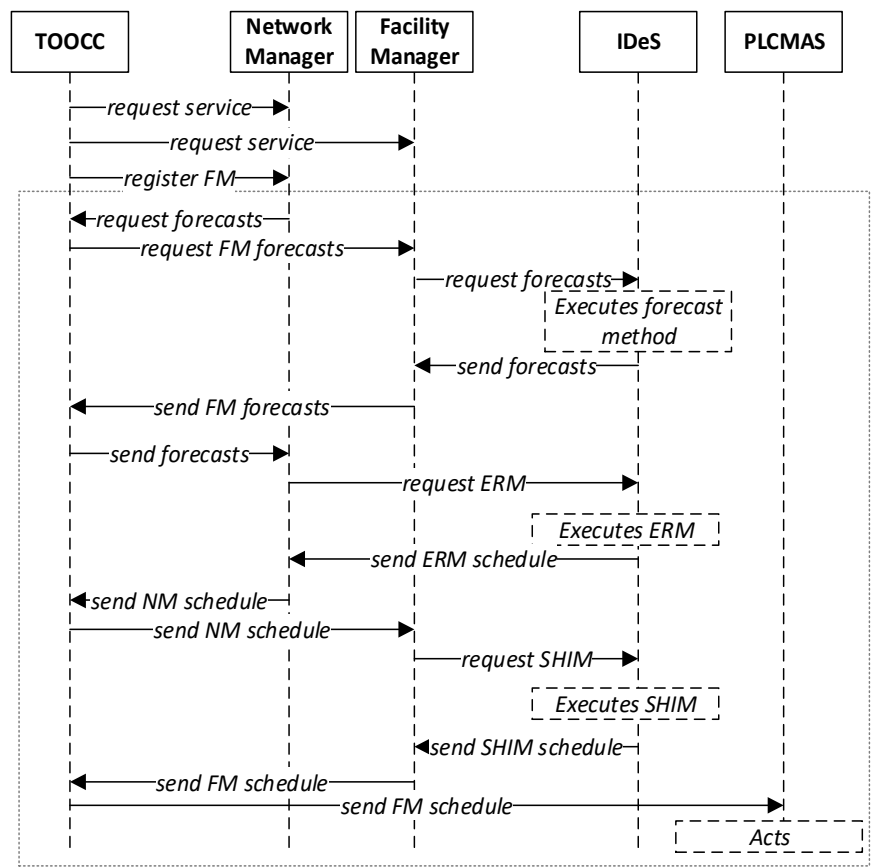

Figure 4. Simplified sequence diagram of the simulation 
Firstly, TOOCC needs to start the interaction with the NM and FM systems. The NM represents the role of microgrid manager, optimizing and managing the consumption and production of all resources connected to its network. Furthermore, it is responsible for ensuring the demand satisfaction, without energy waste. The FM is responsibly for managing the energy resources of a facility. For this, the FM must have information of the consumption, production, characteristics and state of the facility resources (e.g.: sockets, lights, PV generation, etc.).

In order to be able to perform the energy scheduling for all microgrid, NM uses algorithms available in IDeS system, which in this case is the Energy Resources Management (ERM) [10]. ERM is executed in three stages. The first phase is executed in the day before of the desired date, using the collection of the consumption and production forecasts of all entities (e.g.: houses, offices, etc.), which are the network stakeholders, for all hours of the considered day, thereby doing the day-ahead scheduling. In the second phase, the system makes the setup for the hour-ahead scheduling, by using updated, hour-ahead forecasts. Finally, the third phase consists on the real-time scheduling, which works similarly to the previous stage, but considering periods of 5 minutes. Thus, at the beginning of its execution, NM needs the consumption and production's forecasts of all registered facilities. This request is sent to TOOCC, which will send the facilities 'registrations and collect the respective forecasts. One of those facilities will be Load 6 , as a facility.

In order to obtain the forecasts, the FM communicates with the IDeS system, which provides the algorithms for this effect. After its execution, the results are sent to TOOCC, so that the simulation can proceed to the next step, which is the NM execution. When the NM receives the forecasts, it is ready to start the microgrid scheduling. For that purpose, it will request IDeS system for ERM execution.

When the microgrid scheduling is done, the results are sent to TOOCC. In this phase, TOOCC sends the respective result for each registered facility. One of these is Load 6, which is used in this case study as subject example. At this point, Load 6 has all the conditions to perform its own energy scheduling, fulfilling its own goals. For this purpose, the FM sends a request for the SCADA House Intelligent Management (SHIM) platform execution to IDeS system [13]. This platform is able to perform the local residential energy scheduling, considering the demand restrictions provided by the grid management executed by the NM.

At this point, Load 6 has the results of its energy scheduling and it is possible to act directly on its physical devices. In order to do this, the FM sends the results to TOOCC, so that it can order the PLCMAS system to implement the necessary changes to the devices.

\section{Results}

In this section, the obtained results in this process are presented, specifically those related to the consumption and production forecast, the NM's scheduling for Load 6 and the local energy scheduling of Load 6.
Figure 5 presents the forecast of the power demand and solar generation in the microgrid. As it can be seen, the demand of the residential buildings and houses is higher in the morning (between 8am and 11am) and at the end of the day (after $6 \mathrm{pm})$. On the other hand, the office buildings present their higher demand between $8 \mathrm{am}$ and $7 \mathrm{pm}$. The photovoltaic panels show a normal production for a winter day. The peaks of demand are expected at the end of the day, after $6 \mathrm{pm}$ (with exception to $8 \mathrm{am})$, where demand exceeds $80 \mathrm{~kW}$.

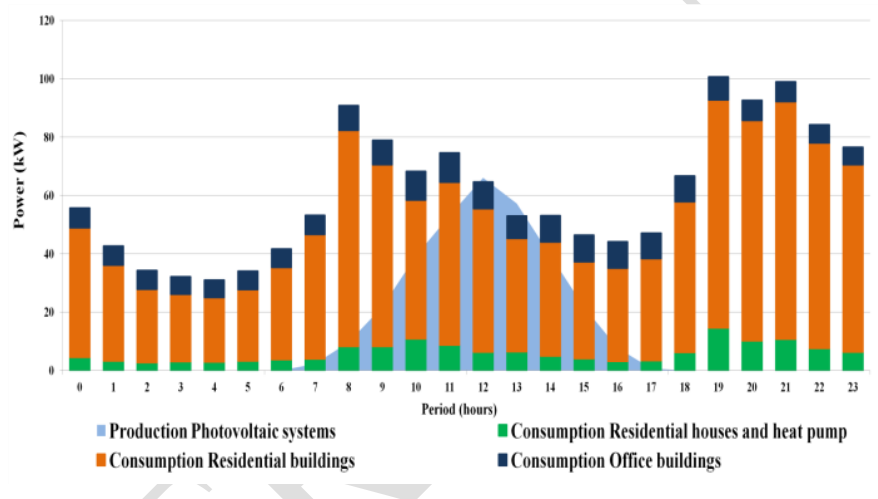

Figure 5. Demand and PV generation forecast of NM

Figure 6 presents the consumption scheduling from the NM to Load 6. As it can be seen in the peak hours, the energy supply suffered a great reduction when compared to the initial forecast, and has been transferred to close periods with a lower demand forecast. For example, the hour between minutes 1205 and 1265, had the highest day-ahead and hour-ahead consumption forecast and therefore suffered a great reduction in consumption (orange in the graph). On the other hand, the previous and the next hour presents a higher consumption than forecasted in order to compensate the mentioned reduction.

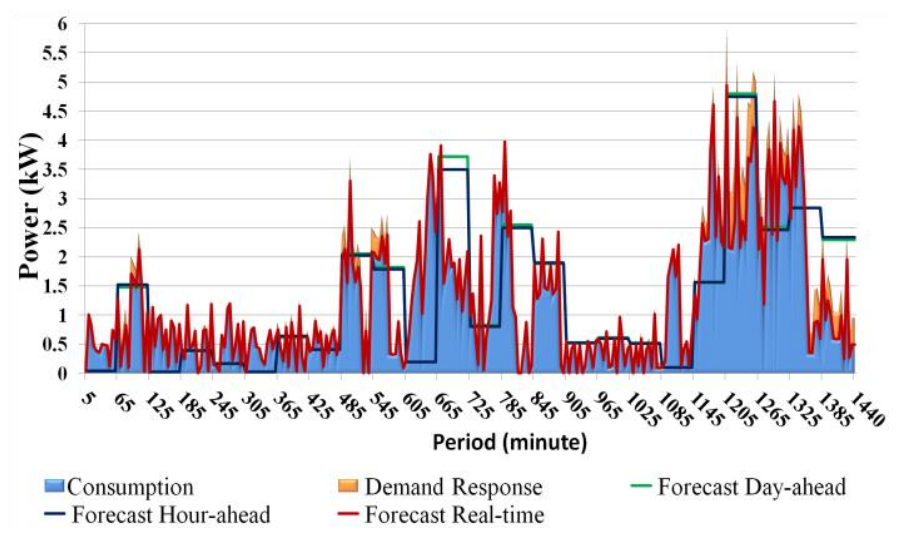

Figure 6. NM scheduling results for Load 6

Figure 7 presents the local scheduling of Load 6, obtained by using the SHIM algorithm, considering the ERM load limit per period. As it is possible to observe, the house optimization was able to meet the ERM limits by adapting its loads consumption. Given that this scenario occurs in the winter season, the HVAC and Water Heater devices, which are very important to fight the cold, had a higher priority in the optimization. The priorities protect the most required loads from cuts as long as the cuts on the other loads are enough to 
meet the ERM load limit. If the scenario occurred in the summer, the priority of Water Heater would be much lower as well as some of the lights, since summer has more daylight hours, thus decreasing the need for as much lights.

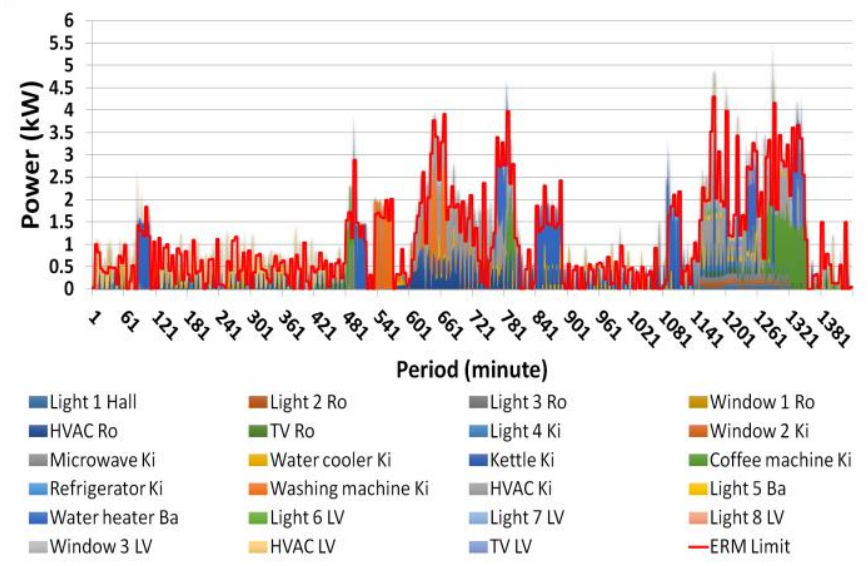

Figure 7. Local scheduling results of Load 6

\section{CONCLUSIONS}

The emergence of Smart Grids has brought a significant change in power systems paradigm, especially due to the large penetration of renewable based generation. Smart Grids perform a key role in current energy markets, taking into account the challenging goals of reducing the use of fossil fuels and energy management. In this scope, analysis and simulation tools allow the participant entities to have mechanisms that allows them to achieve their objectives.

This paper presents TOOCC as a solution to enable interoperability between heterogeneous systems in the energy field. TOOCC works as a centralized facilitator for setting up and launching simulations composed of multiple multi-agent based simulation systems, as well as to provide the independent usage of algorithms and tools for scenarios analysis. The communication between these systems is made using ontologies, thus supporting a common language and enabling all heterogeneous systems to understand each other.

The presented case study has demonstrated TOOCC's scenario creation process and interaction with other systems. This case study is related to energy scheduling of a real 25-bus microgrid, where one of them corresponds to a residence accommodated in the GECAD's laboratory, which performs its own energy resources scheduling.
TOOCC is still under development. Thus, as future work, this tool will be integrated with more systems, algorithms, models and scenarios analysis mechanisms, in order to achieve an increasingly complete tool.

\section{REFERENCES}

[1] R. Wüstenhagen and E. Menichetti, "Strategic choices for renewable energy investment: Conceptual framework and opportunities for further research," Energy Policy, vol. 40, no. 1, pp. 1-10, 2012.

[2] European Commission, "2030 framework for climate and energy policies", 2014, Available: http://ec.europa.eu/clima/policies/2030/index_en.htm [accessed in March 2016]

[3] Lund, H., "Renewable Energy Systems, Renewable Energy Systems A Smart Energy Systems Approach to the Choice and Modeling of 100\% Renewable Solutions", Academic Press, 2nd Edition, May 2014

[4] Pedro Faria, Zita Vale, José Baptista, "Demand Response Programs Design and Use Considering Intensive Penetration of Distributed Generation", Energies, vol. 8, no. 6, pp. 6230-6246, June 2015

[5] S. X. Yang and Y. Wang, "Applying Support Vector Machine Method to Forecast Electricity Consumption"; Computational Intelligence and Security, 2006 International Conference on, Guangzhou, 2006, pp. 929932.

[6] Conejo, A., Carrión, M., and Morales, J. 2010. Decision making under uncertainty in electricity markets., International Series in Operations Research \& Management Science,

[7] Hartog F., Daniele L., Roes J. Toward semantic interoperability of energy using and producing appliances in residential environments. 2015 12th Annual IEEE Consumer Communications and Networking Conference (CCNC). pp. 170-175, January 2015.

[8] Catterson V. et al. An upper ontology for power engineering applications. April 2010. [Online]. Available: http://sites.ieee.org/pesmas/, accessed on July 2016.

[9] Woodridge, M., Jennings, N. R., "Intelligent Agent: Theory and practice", The Knowledge Engineering Review, 10, 2, 115-152, 1995

[10] M. Silva, H. Morais, T. Sousa, P. Faria, and Z. Vale, "Time-horizont distributed energy resources scheduling considering the integration of real-time pricing demand response," PowerTech, 2015 IEEE Eindhoven. pp. 1-6, 2015.

[11] Santos G., Pinto T., Praça I., Vale Z. MASCEM: Optimizing the performance of a multi-agent system. Energy, 111, 513-524, 2016.

[12] Pinto T., Morais H., Sousa T., Sousa T, Vale Z. and Praça I. Adaptive Portfolio Optimization for Multiple Electricity Markets Participation. IEEE Transactions on Neural Networks and Learning Systems, in press, August, 2015.

[13] F. Fernandes, H. Morais, Z. Vale, and C. Ramos, "Dynamic load management in a smart home to participate in demand response events," Energy Build., vol. 82, pp. 592-606, Oct. 2014.

[14] G. Santos, et al., "House Management System with Real and Virtual Resources: Energy Efficiency in Residential Microgrid ", Global Information Infrastructure and Networking Symposium, Porto, 19-21 October, 2016 